\title{
Characterization of Pseudomonas fluorescens Bacteria on Polyurethane using DB-FIB, SEM and STEM
}

\author{
A. R. Blankemeier", H. L. Fraser*, W. Goodson ${ }^{* *}$, D. E. Huber ${ }^{*}$, R. E. A. Williams ${ }^{*}$, H. \\ O. Colijn
}

*Center for the Accelerated Maturation of Materials, Department of Materials Science \& Engineering, The Ohio State University, 2041 College Road, Columbus, Ohio

**Air Force Research Laboratory, Materials \& Manufacturing Directorate, WrightPatterson Air Force Base, Dayton, Ohio

Characterization of biological materials has been challenging due to limitations of current methods such as SEM and confocal microscopy. SEM is useful for topographical information but is still only a two dimensional image. Confocal microscopy is useful for a large sample but does not have the spatial resolution of a TEM or a Dual Beam Focused Ion Beam (DB-FIB). DB-FIB technology has been revolutionary to the field of material science. Traditionally, this approach has been used in metals, ceramics and semiconductor materials but it has been used in a limited way for the characterization of soft materials because it causes degradation of these materials, such as biological materials and polymers. The sample used in this study was a bacterial specimen (Pseudomonas Fluorescens), which forms a Biofilm on a polyurethane paint. The characterization of the interface between an abiotic and biotic material is of interest for surface adherence properties. Surface adherents can cause the fouling of surface coatings or in this case fouling in the paint coating of a surface. If the coating of a surface fails this can lead to corrosion, which can degrade the mechanical properties of the material being protected. In our present studies, the Helios Nanolab 600 DB-FIB was used to create serial sections for 3-dimensional reconstruction and excise thin foils for Transmission Electron Microscopy (Figure1A).

Biofilms were coated with gold to minimize degradation by high-energy ion bombardment. Platinum was deposited to protect the section from $\mathrm{Ga}^{+}$damage while trenching on both sides of the section to be excise. New processes are being developed to reduce the damage to the Biofilm when milled in the DB-FIB. When imaging using the Helios at 350V (Figure2), different surface properties were observed compared with imaging at higher voltages.

Serial sectioning of the Biofilm can provide structural information about the surface adherence mechanisms. Using 2-D images acquired using FEI Slice and View software, a 3-D volume can be reconstructed using software developed at CAMM. The 3-D volume allows for evaluation of the surface adherence mechanisms throughout the entire sample. The problem with this technique is that the damage that is caused to the Biofilm is irreversible. The TEM will offer similar information as well as the spatial variation of the Extracellular Polymeric Substance (EPS) as a function of density. TEM micrographs have shown promise with detailed information about the interface between the Biofilm and paint (Figure1B,C).

References

[1] DJ Stokes, F Morrissey, and BH Lich. Journal of Physics: Conference Series 26 (2006) 50-53. A New Approach to Studying Biological and Soft Materials Using Focused Ion Beam Scanning Electron Microscopy (FIB SEM). 
[2]This work is supported in part by US AFOSR, under STW-21 II program.
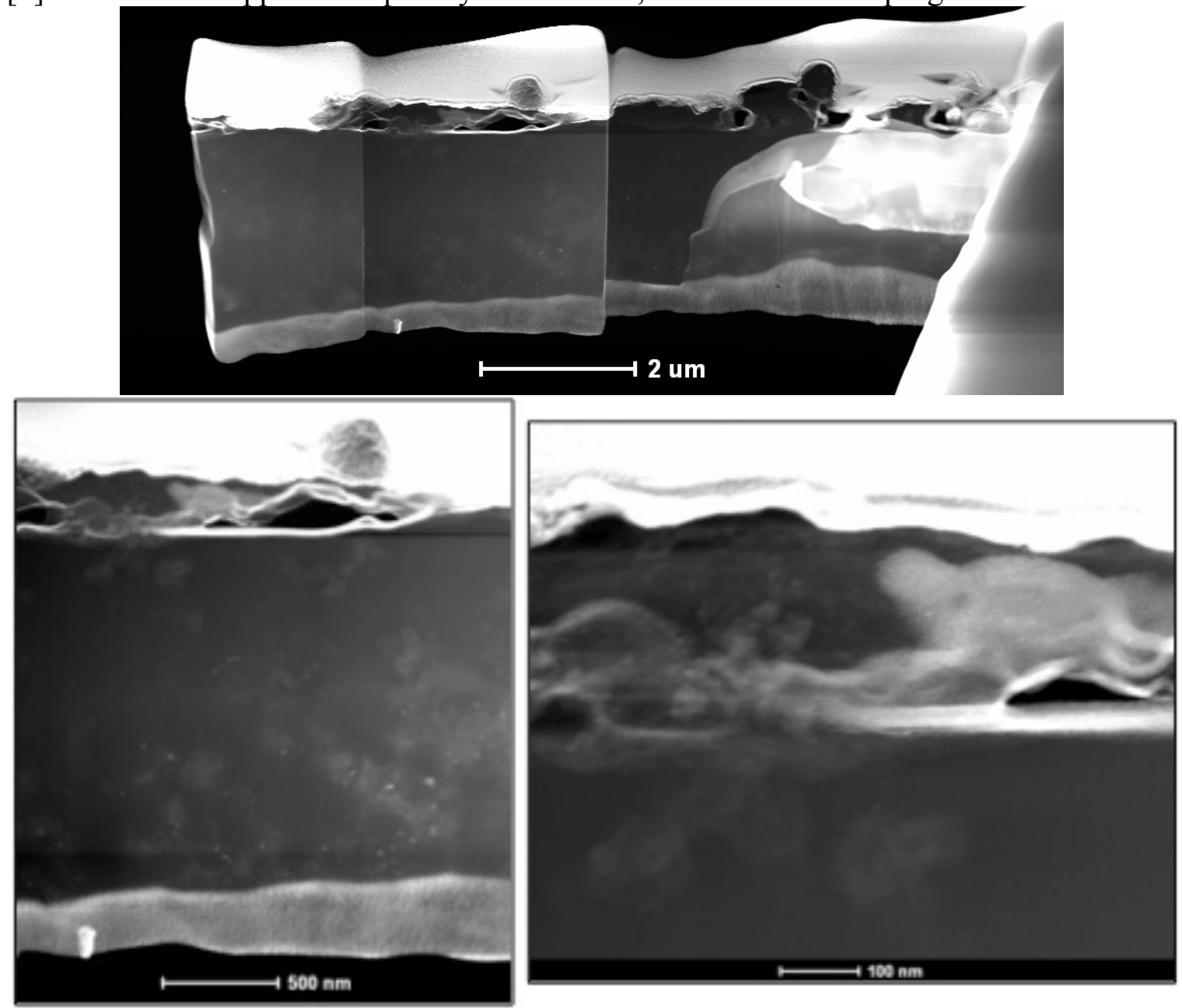

Figure 1. TEM Image A) FIB Foil showing distinct layers of platinum, gold, Biofilm, and Polyurethane Paint w/Carbon black particles B) Biofilm interaction w/polyurethane paint C) Biofilm adherents fouling the surface

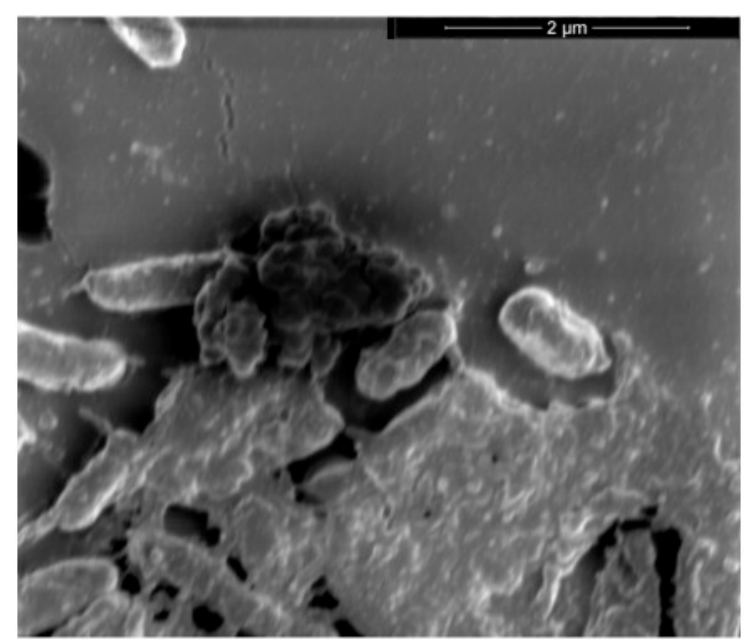

Figure 2. SEM Image at 350V showing Biofilm formation 